\title{
The codon specificity of eubacterial release factors is determined by the sequence and size of the recognition loop
}

DAVID J. YOUNG, CHRISTINA D. EDGAR, ELIZABETH S. POOLE, and WARREN P. TATE

Department of Biochemistry, University of Otago, Dunedin 9054, New Zealand

\begin{abstract}
The two codon-specific eubacterial release factors (RF1: UAA/UAG and RF2: UAA/UGA) have specific tripeptide motifs (PXT/SPF) within an exposed recognition loop shown in recent structures to interact with stop codons during protein synthesis termination. The motifs have been inferred to be critical for codon specificity, but this study shows that they are insufficient to determine specificity alone. Swapping the motifs or the entire loop between factors resulted in a loss of codon recognition rather than a switch of codon specificity. From a study of chimeric eubacterial RF1/RF2 recognition loops and an atypical shorter variant in Caenorhabditis elegans mitochondrial RF1 that lacks the classical tripeptide motif PXT, key determinants throughout the whole loop have been defined. It reveals that more than one configuration of the recognition loop based on specific sequence and size can achieve the same desired codon specificity. This study has provided unexpected insight into why a combination of the two factors is necessary in eubacteria to exclude recognition of UGG as stop.
\end{abstract}

Keywords: release factor; codon recognition; codon specificity; stop codon; translation

\section{INTRODUCTION}

Protein synthesis termination requires recognition of one of three standard stop codons (UAG, UAA, UGA) in the decoding center of the ribosome, followed by hydrolysis of the nascent polypeptide chain from the peptidyl-tRNA in the peptidyl-transferase center (for review, see Poole et al. 2007; Petry et al. 2008; Youngman et al. 2008). Eukaryotes have a single decoding factor, eRF1, recognizing all three standard stop codons (Konecki et al. 1977; Frolova et al. 1994). It is an enigma that eubacteria have retained two decoding RFs with overlapping coding specificities, RF1: UAA/UAG and RF2: UAA/UGA, to achieve the same function (Scolnick et al. 1968). Genetic experiments have identified different tripeptide motifs (PXT in RF1 at residues 188-190, and SPF in RF2 at residues 205-207, Escherichia coli numbering) proposed to determine their different codon specificities (Ito et al. 2000). Structural resolution of the two

Reprint requests to: Warren P. Tate, Department of Biochemistry, University of Otago, P.O. Box 56, Dunedin 9054, New Zealand; e-mail: warren.tate@stonebow.otago.ac.nz; fax: 64-3-479-7866.

Article published online ahead of print. Article and publication date are at http://www.rnajournal.org/cgi/doi/10.1261/rna.2117010. proteins revealed that the motifs comprise part of a recognition loop sequence exposed at the surface of the molecules (Vestergaard et al. 2001; Shin et al. 2004; Graille et al. 2005; Zoldak et al. 2007). Experiments in which 13 amino acids encompassing the recognition loop of RF2 were substituted into RF1 were reported to change codon specificity of the hybrid factor. Surprisingly, this RF1 hybrid was unable to complement an RF2 null E. coli strain, and substituting the equivalent 13 -amino-acid region of RF1 into RF2 in the converse experiment inactivated the RF2 hybrid (Mora et al. 2003). Nevertheless, these results implied that codon specificity of the eubacterial release factors could be switched by exchanging only a small structural region.

Intricate detail as to how the RFs recognize their respective stop codons was revealed through high-resolution crystal structures of RFs bound to the ribosome. They showed that as well as the recognition loop (sometimes referred to as the anticodon loop), a second structural element, the tip of helix $\alpha 5$, contacts the first base of the stop codon (Petry et al. 2005; Korostelev et al. 2008; Laurberg et al. 2008; Weixlbaumer et al. 2008), explaining the remarkable accuracy by which this nucleotide is recognized (Freistroffer et al. 2000). Of the tripeptide motifs within the exposed loop, the third residue 
threonine of the RF1 PXT motif is inferred to form hydrogen bonds through its hydroxyl to both the first and second bases of UAA. Since a second base $\mathrm{G}$ is unable to donate a hydrogen bond to this threonine, which donates its only hydrogen to the first nucleotide $U$, it provides a possible explanation of how RF1 can discriminate between UAG and UGA. In contrast, the first residue of the RF2 SPF motif can recognize both UGA and UAA as the serine hydroxyl can form hydrogen bonds with $A$ or $G$ as the second base of the stop codon. Therefore, within these two RF types, despite their anticodon loops being the same size, there are uniquely different configurations for how the loop achieves recognition and specificity. The structures also revealed that more residues than simply the tripeptide motifs alone make interactions with the stop codons during recognition, implying that they might be important contributors for function and specificity. For example, the side chain of Q185 in E. coli RF1 can form hydrogen bonds with both A or $\mathrm{G}$ at the third position of the stop codon, whereas RF2 has a valine that precludes recognition of $\mathrm{G}$. In this case, recognition is restricted to A alone at the third position of the stop codon.

Bioinformatic analyses have identified 15 specificitydetermining positions in both the recognition loop and helix $\alpha 5$ regions of RF1 and RF2 (Oparina et al. 2005). These positions comprise differentially conserved residues that are proposed to account for the differences in functional specificity between the two factors. Interestingly, six of these positions are contained within the 13 amino acids that were transferred between RF1 and RF2 in loop exchanges (Mora et al. 2003).

Mitochondria have eubacterial-like RFs consistent with their originating from an endosymbiotic $\alpha$-proteobacterial ancestor. In most organisms mitochondria use only two stop codons, UAG and UAA, and their genomes contain only a single RF1-type mitochondrial gene (MRF1). They lack an RF2-type factor that could recognize UGA. An example is Saccharomyces cerevisiae MRF1, which exhibits high sequence similarity to eubacterial RFs and particularly RF1 (Pel et al. 1992). It contains a typical RF1-type PXT motif, and in an in vitro release assay responds to only UAA and UAG, indicating that it is a genuine RF1 homolog (AskarianAmiri et al. 2000).

In this study, we deconstructed the recognition loop of the RFs to examine its role in codon recognition and specificity. We have determined that the tripeptide motif alone is not sufficient to determine specificity, but rather a number of residues within the 10-amino-acid loop and adjoining region are critically important. A shorter loop can be compensated for by specific changes to the loop sequence to achieve the same highly restricted codon specificity. The study provides key insights into why two different factors have been retained to recognize the three standard stop codons in eubacteria, in stark contrast to how eukaryotes achieve the same critical biological function.

\section{RESULTS}

\section{What residues of the recognition loop determine stop codon specificity?}

We have named the major structural feature of eubacterial decoding factors involved in stop codon recognition the "recognition loop" (referred to as the "anticodon loop" in previous publications). It contains the PXT (RF1) and SPF (RF2) motifs and typically comprises 10 amino acids (Fig. 1A). The study in which 13 residues from RF2 were substituted into RF1 did not address whether the SPF tripeptide alone could achieve a recognition switch (Mora et al. 2003). The three residues additional to the loop residues included in the exchange in this study form part of strand $\beta 4$, and two are specificity-determining positions that distinguish the RF types (numbered in Fig. 1A). Three of the 10 loop residues are common to both factors, and four others (including the tripeptide motif) have been identified as specificitydetermining positions (Oparina et al. 2005).

To determine the minimum number of residues and their positions required to switch the codon specificity of RF1 to that of RF2, a series of RF1 variants was constructed with successive changes to the recognition loop that incrementally converted it to that of RF2 (Fig. 1B). The RF1 variants were tested for codon-dependent peptidyl-tRNA hydrolysis activity in vitro using the simple stop codonactivated release assay (Tate and Caskey 1990), and then in vivo using a translation termination assay based on multiple domains of Staphylococcus aureus protein A as a reporter gene (Bjornsson et al. 1998; Cridge et al. 2006).

The in vitro release activities of the variant RF1 proteins were tested with all three standard stop codons, and with the near-cognate (2/3) sense codon, UGG (selected variants [Fig. 1C] and full panel of variants [Supplemental Fig. 1A]). All six of the RF1 loop variants showed a significant loss of release activity compared to that of wild-type RF1 with both cognate stop codons. Significantly, the RF1 variant where the motif PAT alone was substituted with SPF (RF1-SPF) did not function with any of the stop codons, suggesting that the SPF motif by itself could not switch the codon specificity of RF1 to that of RF2. In contrast, if the RF1 specificity-determining glutamate immediately following the tripeptide motif was substituted with aspartic acid in addition to SPF as in RF2 [RF1-SPF(D)], there was a small partial restoration of release activity. Interestingly, however, this was not a simple specificity switch. As well as still recognizing the RF1 cognate stop codons UAG and UAA, the RF1-SPF(D) variant showed a significant increase in release activity at both the noncognate stop codon UGA and the sense codon UGG when compared with wild-type RF1. Further changes to the recognition loop resulted in a return to a complete loss of activity [shown only with RF1-(K)SPFD and RF1-RF2 recognition loops in Fig. 1C and more fully in Supplemental Fig. 1A]. Surprisingly, in our experiments, even substituting 
A

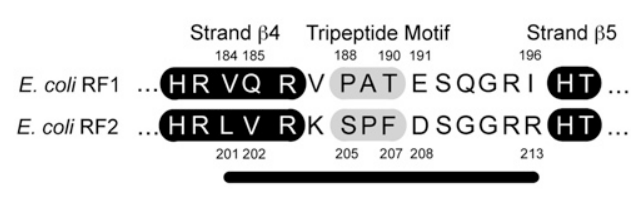

B

RF1-SPF

RF1-SPF(D)

RF1-(K)SPFD

RF1-QtoG

RF1-ItoR

RF1-RF2 rl

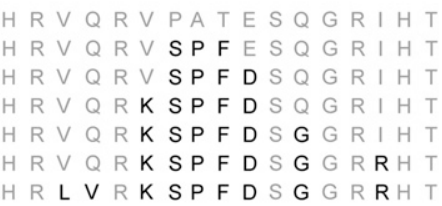

H R V Q R V PATESQ GR I H T

H R L V R K SPFDSG GRRH T

C

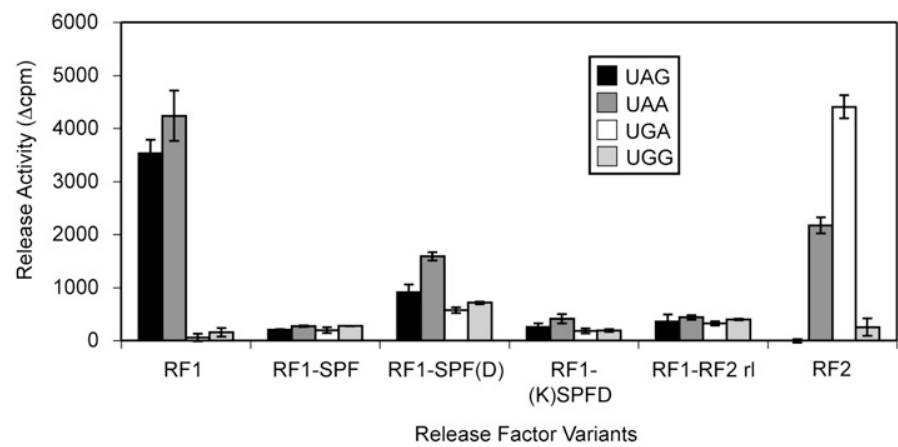

D

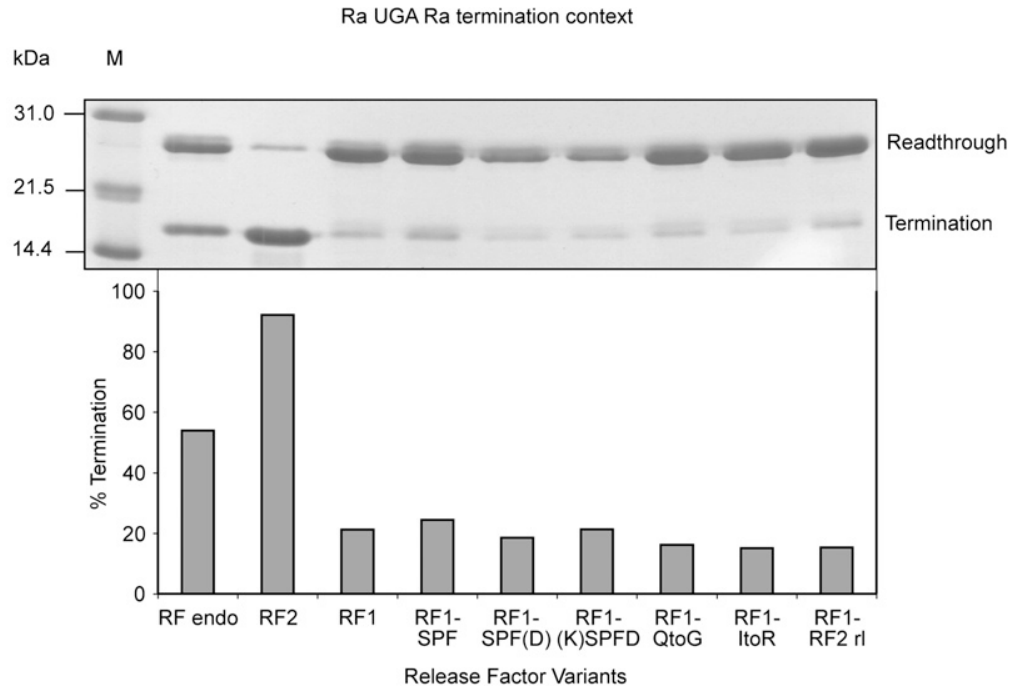

FIGURE 1. RF recognition loop codon specificity determinants. (A) The recognition loop regions of E. coli RF1 and RF2. The 10 loop residues are shown with the $\beta$-strands surrounding the recognition loop highlighted in black. The tripeptide motifs (PXT/SPF) are highlighted in gray. Specificity-determining positions are numbered, and the black line indicates the amino acids analyzed. (B) The RF1-RF2 recognition loop variants. The residues substituted are shown in black. $(C)$ The codon-dependent peptidyl-tRNA hydrolysis activities of the RF1-RF2 recognition loop variants at the standard stop codons UAG, UAA, and UGA, and the sense codon UGG. Release activity is given in $\Delta$ cpm of [ ${ }^{3} \mathrm{H}$ ] fMet released. The RF variants were assayed in triplicate with each experiment repeated twice to ensure consistent results between assays. RF2 is included as a positive control for UGA activity. The error bars represent the standard deviation of the mean. $(D)$ The effect of overexpression of the RF1-RF2 recognition loop variants on termination efficiency at the Ra UGA Ra termination context in the E. coli suppressor strain CDJ64. (Top) A SDS polyacrylamide gel showing readthrough and termination products that reflect termination efficiencies. (Bottom) The calculated termination efficiencies derived from this gel experiment. (M) Marker; (RF endo) endogenous RFs.

the whole extended 13-residue recognition loop region of RF2 into RF1 did not switch stop codon specificity but also resulted in an inactive factor.

The " $3 \mathrm{~A}^{\prime}$ translation termination assay" (Bjornsson et al. 1998; Cridge et al. 2006) was used to test whether the results with the RF1 recognition loop variants in vitro could be reproduced in vivo in the normal physiological envi- ronment of the bacterial cell with all of the translational components present. In this assay, the stop codon is placed in a "rare" sequence context that has a high readthrough frequency when in competition with a suppressor tRNA. We showed previously that overexpressing cognate RF in vivo significantly increases termination efficiency at such contexts, whereas the noncognate factor (presumably because 
of nonproductive competition for the ribosomal site) actually decreases termination efficiency (Cridge et al. 2006). If a variant RF is overexpressed that is capable of recognizing the stop codon and promoting peptidyl-tRNA hydrolysis in vivo, then there will be an observable increase in termination efficiency. On the other hand, a decrease indicates that the overexpressed factor competes with the endogenous factors for the decoding site but is inactive for releasing a product. To test for a change in codon specificity of the RF1 variants, we used a rare ( $\mathrm{Ra}$ ) RF2-specific UGA termination context $(\mathrm{Ra}$ UGA Ra) separating domains two and three of the threedomain reporter gene in the E. coli CDJ64 UGA suppressor strain. As a control for retention of original codon specificity, an RF1-specific UAG termination context (Ra UAG Ra) was used in the E. coli XA102 UAG suppressor strain.

Termination results in a two-domain protein product, and readthrough a three-domain product. The termination rate (\%) was determined after normalization of the band intensity. With endogenous RF2, termination efficiency in competition with a suppressor tRNA in the CDJ64 UGA suppressor strain was $\sim 55 \%$ at the Ra UGA Ra context (Fig. 1D, RF endo). Endogenous RF1 gave a termination efficiency of $\sim 60 \%$ at the Ra UAG Ra context in the XA102 UAG suppressor strain (Supplemental Fig. 1B). Overexpression of RF2 (as a positive control) at the Ra UGA Ra context increased termination efficiency markedly from $\sim 55 \%$ to $\sim 90 \%$, whereas expression of noncognate RF1 significantly depressed it to $\sim 20 \%$ (Fig. 1D). Overexpressed RF1 and RF2 had the reverse effects as expected with the Ra UAG Ra context (Supplemental Fig. 1B). The six RF1 recognition loop variants all gave similarly decreased termination efficiency compared to vector alone at the UGA or UAG termination contexts. Hence, none of the variants recognized UGA or UAG productively as a stop codon to enhance competition with the suppression. We infer that this was because changes to the recognition loop inactivated the factor's ability to release the protein. Overall, the results were consistent with those of the in vitro assay.

Importantly, these results showed that substituting the tripeptide motif or, indeed, the complete exposed loop (together with three residues of the $\beta 4$-sheet of RF2) into RF1 did not swap the factor's codon specificity either in vitro or in vivo.

\section{Are specific combinations of residues essential for stop codon specificity?}

Although the RF1-SPF(D) variant showed partial release activity in vitro (Fig. 1C), one more substitution ( $\mathrm{V}$ to $\mathrm{K}$ at the residue immediately preceding it) resulted again in a complete loss of activity. While this residue has not been previously identified as a specificity-determining position, only a restricted number of amino acids are tolerated at this site in eubacterial RFs. The RF2 lysine is never observed in any RF1 and may be incompatible with functional RF1 decoding. Introducing inactivating substitutions like this into the loop masks potential contributions of other residues in the subsequent RF1 variants. To circumvent this, another series of RF1 variants was constructed starting with the partially active RF1-SPF(D) variant but making individual rather than successive substitutions within the recognition loop (Fig. 2A).

The in vitro codon-dependent peptidyl-tRNA hydrolysis activity revealed no release activity for the RF1-(VQtoLV)SPFD variant. This highlights the importance of Q185 for RF1 activity (Fig. 2B). In contrast, the ${ }^{196} \mathrm{I}$ to $\mathrm{R}$ change in the RF1SPFD(ItoR) variant allowed increased recognition of the noncognate stop codon UGA and the near-cognate sense codon UGG, but not, as shown in Figure 2C, at other nearcognate sense codons starting with U (UGC, UGU, UCA, UUA) or with AAG or AGA. The RF1-SPFD(ItoR) variant's codon specificity pattern was similar to that observed for

A

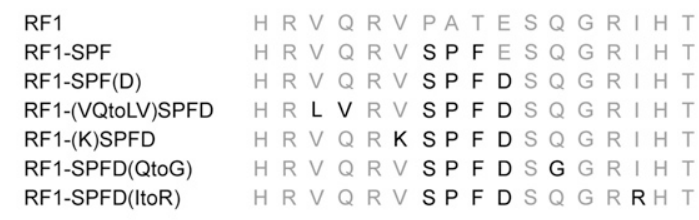

B

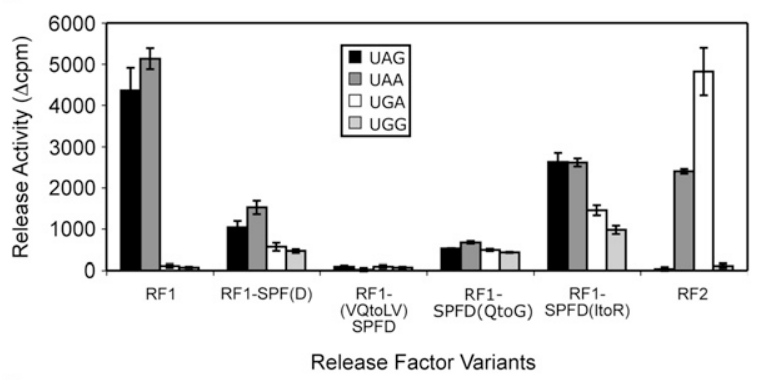

C

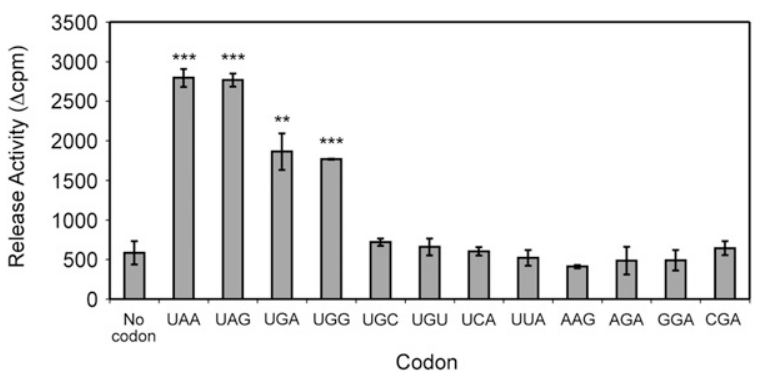

FIGURE 2. RF1 recognition loop residue substitutions affect codon recognition and specificity. (A) The RF1 SPFD recognition loop variants. Substitutions are shown in black. $(B)$ The codon-dependent peptidyl-tRNA hydrolysis activities of the SPFD recognition loop variants at the standard stop codons UAG, UAA, and UGA, and the sense codon UGG. The RF variants were assayed and the data analyzed as described for Figure 1C. (C) Codon specificity of the RF1 SPFD(ItoR) recognition loop. The SPFD(ItoR) variant was assayed with the codons indicated and the data analyzed as for Figure 1C. Statistical significances, $\left.\left.{ }^{* *}\right) P<0.01,{ }^{* * *}\right) P<0.001$ (Student's twotailed $t$-test), were determined by comparison to the activity of RF1SPFD(ItoR) with no codon. 
RF1-SPF(D) but enhanced at all four codons, UAA, UAG, UGA, and UGG. Significantly, these variants [and SPFD(QtoG), Supplemental Fig. 2] recognized not only all three stop codons but also the sense codon UGG.

The effect of overexpression of the initial series of RF1 recognition loop variants on in vivo termination efficiency at the two termination contexts revealed that all six decreased termination efficiency at the Ra UGA Ra termination context similar to that observed for RF1 (Fig. 1D). As well, they were no longer functional at the Ra UAG Ra termination context (Supplemental Fig. 1B). This suggested that none of the RF1 recognition loop variants with further RF2 substitutions had acquired competitive UGA recognition after losing UAG recognition. In contrast, the in vivo termination efficiencies after overexpression of RF1-SPFD(QtoG) and RF1-SPFD(ItoR) from the second series were decreased less markedly compared with the other inactive variants, suggesting that they had retained some activity in vivo (data not shown) consistent with the in vitro release assays.

These experiments reveal that the collective identities of the specific amino acids at a number of positions throughout the recognition loop are critically important for both codon recognition and codon specificity.

\section{Novel recognition loops in nematode MRF1 proteins}

An alignment of the known and predicted nonvertebrate MRF1 protein sequences (Fig. 3A) showed that the MRF1s of three nematode species-Caenorhabditis elegans, Caenorhabditis briggsae, and Brugia malayi-each has unique recognition loops compared to other MRF1 and eubacterial RF1 proteins. In C. elegans and C. briggsae MRF1 proteins, the loops are two amino acids shorter than those of all other classical RF1-type factors, and there is variation in the normally invariant RF1-specific motif PXT(E), with $\mathrm{N}$ for $\mathrm{T}$ in the third position of the motif, and $\mathrm{D}$ instead of $\mathrm{E}$ for the following specificity-determining position (Fig. 3B, top). In contrast, the B. malayi loop is constructed somewhat differently with a single amino acid deletion. However, despite their unique recognition loops, the nematode MRF1 proteins have high sequence homology with classical MRF1s in their structural domains.

\section{The shorter MRF1 recognition loop recognizes UAG and UAA}

To test whether the unusual C. elegans MRF1 loop supported the same codon specificity as the other RF1-type

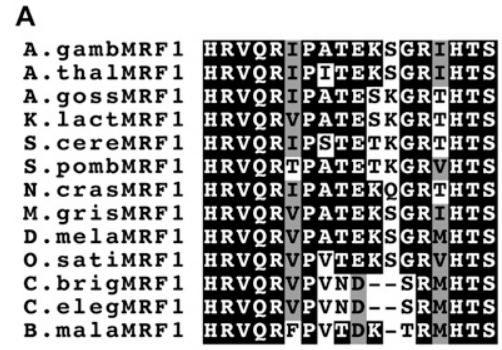

C

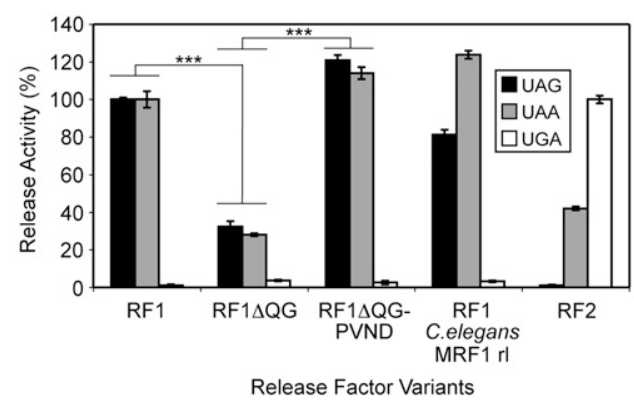

B

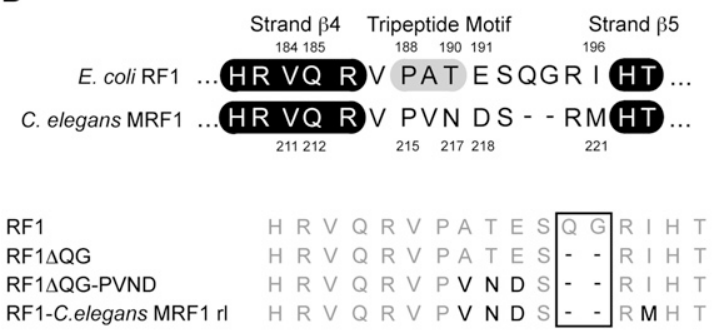

D

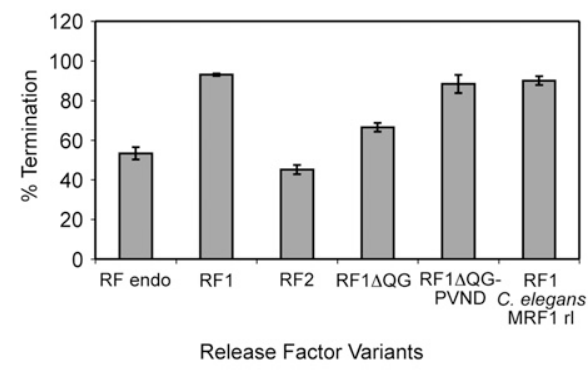

FIGURE 3. Codon recognition and specificity of the C. elegans MRF1 novel recognition loop. (A) A sequence alignment of the recognition loop regions of a selection of nonvertebrate MRF1 proteins. Identical amino acids are white on a black background, those similar are black on a gray background, and different amino acids are black on a white background. Full listings for each species are in Supplemental Table 1. (B, top) The recognition loop regions of E. coli RF1 and C. elegans MRF1. The $\beta$-strands surrounding the recognition loop are shown in black. The RF1specific tripeptide (PXT) motif of E. coli RF1 is highlighted in gray. Specificity-determining positions are numbered. (Bottom) The RF1 C. elegans MRF1 recognition loop variants. Amino acid substitutions are shown in black, and deletions are boxed. (C) The codon-dependent peptidyl-tRNA hydrolysis activities of the RF1 C. elegans MRF1 recognition loop variants at the standard stop codons UAG, UAA, and UGA. The release activities are expressed as percentages of the release activity of RF1 for UAG and UAA, and RF2 for UGA. The RF variants were assayed and data analyzed as for Figure 1C. $(D)$ The effect of overexpression of the RF1 C. elegans MRF1 anticodon loop variants on termination efficiency at the Ra UAG Ra termination context in the E. coli suppressor strain XA102. The RF variants were assayed in triplicate in three separate experiments to ensure consistent results between assays. The error bars represent the standard deviation of the mean. Statistical significances, $\left(^{\star * *}\right) P<0.001$ (Student's two-tailed $t$-test), were determined by comparison to the activity of either RF1 or RF1 $\Delta$ QG. 
proteins, a series of E. coli RF1 variants was constructed with successive changes that converted the recognition loop incrementally from that of E. coli RF1 to that of the $C$. elegans MRF1 loop (Fig. 3B, bottom). The in vitro peptidyltRNA hydrolysis activities revealed that the deletion variant, RF1 $\Delta$ QG, missing two amino acids like the C. elegans MRF1 loop but retaining the other RF1 residues, supported codon recognition activity but at a reduced level ( $\sim 30 \%$ of RF1 for both UAA and UAG) (Fig. 3C). There was no change in specificity. The RF1 $\Delta$ QG-PVND variant, wherein the classic E. coli PAT(E) sequence was changed to the $C$. elegans PVN(D), had its release activity restored and enhanced to above wild-type levels $(\sim 120 \%$ for both UAG and UAA compared with E. coli RF1). Interestingly, the variant with the complete loop of $C$. elegans MRF1 now showed a preference for recognition of UAA over UAG (ratio 3:2) compared with E. coli RF1 (ratio 1:1). In the $C$. elegans mitochondrial genome, UAA is used almost exclusively with UAG used only once (Okimoto et al. 1992).

The RF1 C. elegans MRF1 recognition loop variants were also tested in vivo for their decoding activity at the Ra UAG Ra termination context (Fig. 3D). The termination efficiency with the overexpressed RF1 $1 \Delta \mathrm{QG}$ variant was intermediate between that found for the endogenous factor alone and overexpressed RF1, suggesting that while it is competitive, it is not as active as E. coli RF1. Overexpression of both RF1 $\Delta$ QGPVND and the complete loop substitution variant (RF1-C. elegans MRF1 rl) resulted in termination efficiency comparable to the wild-type RF1 loop sequence. As the uniquely engineered $C$. elegans MRF1 recognition loop (in the $E$. coli RF1 chimera) clearly supported UAG and UAA codon specificity both in vitro and in vivo, there is strong evidence that functional recognition loops of RF proteins can be constructed with different templates to retain recognition of the standard cognate stop codons.

\section{What residues of the PVND sequence are important for restoring release activity?}

As shown above, changing three residues including two in the tripeptide motif and the residue following (PATE to PVND) in the recognition loop restored the RF1 loss of catalytic activity incurred as a consequence of removing two amino acids to simulate the size of the C. elegans loop. To investigate whether the identities of all three amino acids were important for restoring activ- ity, two single variants, RF1 $\Delta \mathrm{QG}-\mathrm{PA}(\mathrm{N}) \mathrm{E}$ and RF1 $\Delta \mathrm{QG}-$ $\mathrm{PAT}(\mathrm{D})$, and the double variant, RF1 $\Delta \mathrm{QG}-\mathrm{PA}(\mathrm{ND})$, were constructed (Fig. 4A, top). The third single variant, $\mathrm{V}$ for A, was not made as several residues are allowed in this position in the classical PXT tripeptide motif (Ito et al. 2000).

The two single variants, RF1 $1 \Delta \mathrm{QG}-\mathrm{PA}(\mathrm{N}) \mathrm{E}$ and RF1 $1 \Delta \mathrm{QG}-$ PAT(D), did not compensate for the lowered in vitro activity of this deletion variant (Fig. 4A, middle). On the other hand, the double variant with both changes showed a restoration of activity to near that of wild type ( $\sim 80 \%$ of wild type) and did not change its specificity. The other sequences of the $C$. elegans loop further enhanced the effect. In vivo overexpression of the deletion variant, RF1 $\Delta \mathrm{QG}$, and the double substituted variant, RF1 1 QG-PA(ND), at the Ra UAG Ra termination context gave lowered and restored termination efficiencies, respectively, consistent with the in vitro studies (Fig. 4A, bottom). The single substitution variants RF1 $\Delta$ QG$\mathrm{PA}(\mathrm{N}) \mathrm{E}$ and RF1 $\Delta \mathrm{QG}-\mathrm{PAT}(\mathrm{D})$ gave activities similar to the deletion variant $\mathrm{RF} 1 \Delta \mathrm{QG}$ alone.

A second series of RF1 variants without the QG deletion was created in which PATE was progressively changed to PVND (Fig. 4B, top). Surprisingly, the in vitro codondependent peptidyl-tRNA hydrolysis activities of these variants showed that substitution of the RF1-specific E to $\mathrm{D}$ in the last position [RF1-PAT(D)] enhanced recognition
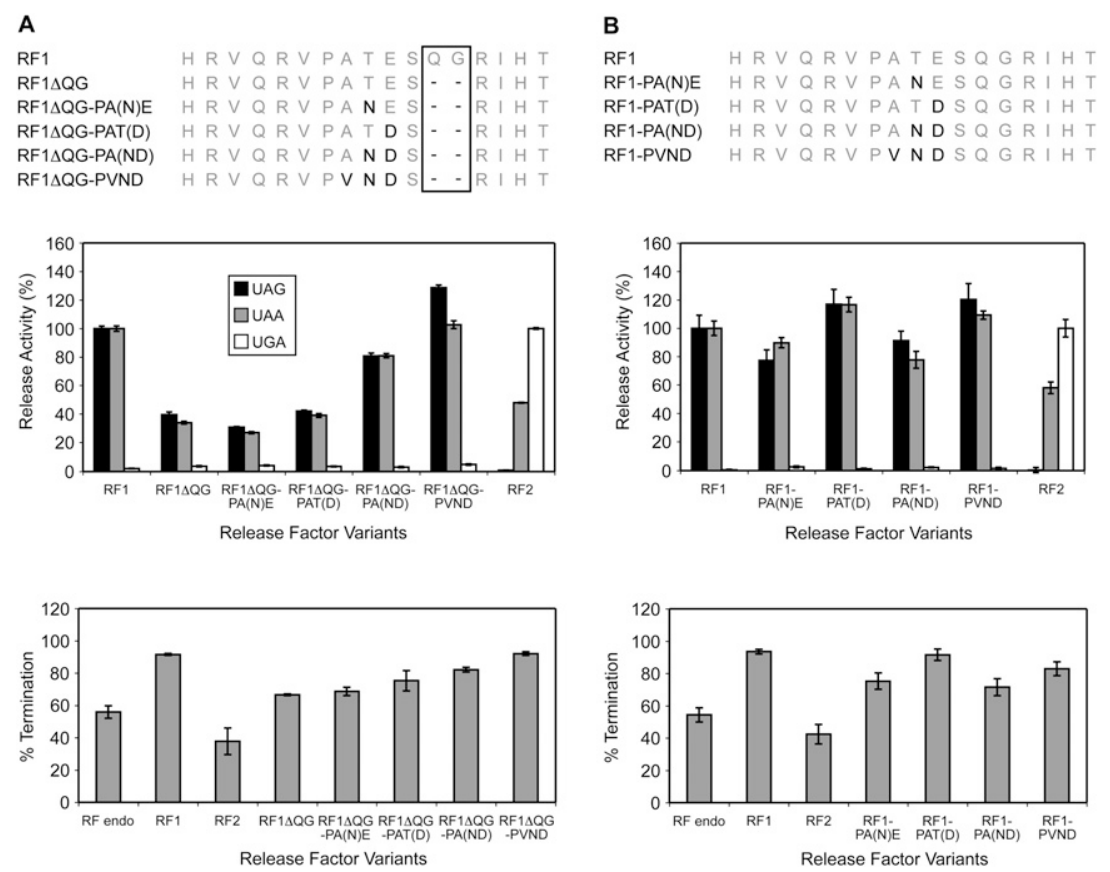

FIGURE 4. The activity of PVND motif variants in the RF1 recognition loop. (Top) The RF1 PVND variants with shortened loop size as for C. elegans $(A)$ or as for a typical RF $(B)$. Amino acid substitutions are shown in black. (Middle) The codon-dependent peptidyl-tRNA hydrolysis activities of the RF1 PVND motif variants at the standard stop codons UAG, UAA, and UGA. The release activities are expressed as percentages of the release activity of RF1 for UAG and UAA, and RF2 for UGA. The RF variants were assayed and data analyzed as for Figure 1C. (Bottom) The effect of overexpression of the RF1 PVND variants on termination efficiency at the Ra UAG Ra termination context in the E. coli suppressor strain XA102. Replicates and analyses were as for Figure 3D. 
activity by $\sim 20 \%$ with UAG and UAA compared with wildtype E. coli RF1 $\sim 100 \%$ (Fig. $4 \mathrm{~B}$, middle). This was also reflected in the PVND double variant. The other partial substitutions [RF1-PA(N)E and RF1-PA(ND)] somewhat depressed activity $(\sim 80 \%-90 \%$ of wild type). All variants retained strict stop codon specificity. In vivo, all four variants showed an increase in termination efficiency at the UAG termination context compared with endogenous RFs (Fig. 4B, bottom). The order of effects replicated what was seen for the in vitro release assays. Together, these results showed that the PVND motif, even though it is extremely rare, still functions well in a full-length RF1 recognition loop. It highlights that despite the highly conserved RF1-specific PXT motif, alternative sequence configurations in a typical 10-residue loop can be accommodated without loss of specificity.

Importantly, together these results showed that the unusual shortened C. elegans recognition loop had the ability to recognize stop codons with high efficiency and with specificity mainly through the four amino acids within the PVND motif functioning cooperatively, but surrounding residues were also important for functional activity.

\section{DISCUSSION}

\section{Key structural features of the RFs and stop codon specificity}

Two questions must be answered to understand why there are two different factors in prokaryotes for recognition of the three standard stop codons UAA, UAG, and UGA: (1) how do the factors restrict recognition to only three of the 64 codons; and (2) how are A and G at the second and the third bases of these codons distinguished?

The high-resolution structures of termination complexes have shown that restriction of recognition to purines only in the second and third codon positions is a function of the recognition loop (Korostelev et al. 2008; Laurberg et al. 2008; Weixlbaumer et al. 2008). The location of the restricted $\mathrm{U}$ for the first position was unexpectedly indicated in the earlier ribosomal termination complex structures (Petry et al. 2005) to be close to the helix $\alpha 5$ region, a part of the RF structure that has the same sequence in both RF1 and RF2. This small region has been confirmed in biochemical studies to be in close contact with this base (DJ Young, ES Poole, and WP Tate, unpubl.). Eubacteria over a wide phylogeny have maintained two RFs to share recognition of the three stop codons, in sharp contrast to the single RF of the archaea and eukaryotes. Our detailed analysis of the recognition loop has provided insight into this intriguing biological enigma.

\section{A tuning model for stop codon recognition by RFs}

The substitution of the tripeptide motif or the complete recognition loop of RF1 with the equivalent from RF2 simply led to an inactive factor in our experiments when analyzed both in vitro and in vivo. The previous assumption that there was a small discrete isolated region (in the recognition loop and tip of helix $\alpha 5$ ), functionally similar to the anticodon of a tRNA, that is alone responsible for determining codon specificity is clearly too simplistic. Our data imply that RFs do not function with the body of the factor simply acting as a generic adapter that connects the recognition loop of domain 2 to the GGQ hydrolysis-inducing motif (common to RF1 and RF2, and even to the structurally dissimilar eukaryotic factor, eRF1) in domain 3.

Even for tRNAs, anomalies of the adapter hypothesis (Crick 1958) have led to a tuning model being proposed (Dale and Uhlenbeck 2005; Ledoux and Uhlenbeck 2008). It suggests that each tRNA has evolved to adjust its affinity for EF-Tu and for the ribosome in a way that compensates for the particular affinity of its cognate amino acid and anticodon. As a result, tRNAs may also not be generic interchangeable adapters but have been individually crafted and tuned so that all are translated uniformly.

Could RFs be structurally tuned to balance stop codon specificity and efficiency in an analogous manner so that translation of stop codons occurs within a narrow efficiency range? Substituted residues that have been swapped between factors may have structural clashes with the rest of the protein. For example, ${ }^{201 / 202} \mathrm{LV}$ and ${ }^{204} \mathrm{~K}$ of RF2 may not be able to replace ${ }^{184 / 185} \mathrm{VQ}$ and ${ }^{187} \mathrm{~V}$ of RF1, a conclusion supported by the high-resolution structures. An RF2 loop on an RF1 framework may not pack optimally onto the stop codon due to these consequential clashes with residues on the RF1 structure. A Van der Waals surface representation of native RF1 bound to the stop codon in the decoding center showed close packing of the stop codon with surrounding elements of RF1 and 16S rRNA (Laurberg et al. 2008).

Shortened recognition loops, while rare naturally, are allowable as present in C. elegans mtRF1. In the highresolution structure of RF1 bound to the ribosome, neither of the two residues missing in the $C$. elegans loop contacts the UAA stop codon (Laurberg et al. 2008), but their absence is likely to alter the loop's conformation and change the orientation of residues contacting the stop codon. Nevertheless, we have shown that an E. coli RF1 loop lacking the two missing residues of the $C$. elegans mtRF1 loop retains some activity, and, furthermore, the hybrid factor can be restored to full activity by additional sequence variations, a form of tuning within the recognition loop itself. This implies that not only are the recognition loops of the RF1 and RF2 structures tuned to avoid structural clashes with the body of the protein, but also the loop itself is tuned through its sequence for optimal orientation toward the stop codon.

\section{How can our results be aligned with the previous concept of key specificity-determining tripeptide components within exchangeable recognition loops?}

RF2 codon-specific activity could not be rescued in vivo from the swap of the RF2 loop into RF1, compatible with 
our findings. A puzzling inconsistency, with which our data are in conflict, was that in vitro this same hybrid had switched codon specificity. In contrast, the RF1 loop could not be swapped for that of RF2 and retain activity in vitro (Mora et al. 2003).

A tripeptide motif in the two RFs, subsequently shown to be within their recognition loops (Vestergaard et al. 2001; Shin et al. 2004; Graille et al. 2005; Zoldak et al. 2007), was highlighted as the likely determinant for how UAG and UGA were distinguished at the second and third positions (Ito et al. 2000). The high-resolution structures (Korostelev et al. 2008; Laurberg et al. 2008; Weixlbaumer et al. 2008) showed that the motifs form interactions with the first two bases of the stop codon rather than the second and third, and, therefore, they should not be the sole determinants of specificity. Our study showed that substituting the tripeptide alone, indeed, did not change specificity but resulted in the loss of all activity, either when assayed for function in vitro or in the intact bacterial cell in vivo.

How can we accommodate the apparent incompatibility of our data with the important original genetic experiments of Ito et al. (2000) showing switched specificity? We believe that in reality the two sets of studies are not incompatible despite some differences in the conclusions drawn. The genetic experiments highlighted a small region (the $\mathrm{D}$ region) encoding 15 amino acids within the RF genes as responsible for specificity, and this region not only contained the important tripeptide motif residues but also highlighted the importance of ${ }^{185} \mathrm{Q} /{ }^{202} \mathrm{~V}$ and ${ }^{196} \mathrm{I} / \mathrm{R}$ (Nakamura and Ito 2002). Other residues were deemed to be not so critical, but this may be because a number of different substitutions were allowable at those sites without causing structural clashes even though the specific residue found there in the other factor might not be compatible. Some nucleotide changes (and hence amino acid substitutions such as replacing the ${ }^{185} \mathrm{Q}$ in RF1 with the equivalent position $\mathrm{V}$ in RF2) were made to the region for their "swapping cassette" for ease of cloning. Such a change would have supported a more relaxed specificity for codon recognition at the third base. Whether the tripeptide "swaps" (along with the other indirect amino acid changes) that switched recognition between UAG and UGA had broader specificity was not reported (Ito et al. 2000). Of interest in this study, also, was that $\mathrm{N}$ was an acceptable replacement for the very highly conserved $\mathrm{T}$ of the RF1 tripeptide motif PXT. This substitution (PVN) is found, however, in the C. elegans MRF1 loop and was also functional in our studies with the E. coli RF1 loop. Presumably, like the hydroxyl of the T in the PXT motif, $\mathrm{Q}$ is able to form hydrogen bonds to the first and second bases, with appropriate structural tuning.

A larger substitution (tetrapeptide) that included the adjacent highly conserved aspartate from the RF2 loop did restore a low level of activity but not with switched specificity, rather it conferred loosened specificity (the three standard stop codons were recognized and as well the near-cognate sense codon UGG). Interestingly, the insertion of RF2's ${ }^{208} \mathrm{D}$ alone into the RF1 loop at position 191 enhanced RF1's codon recognition (see Fig. 4B). Why then has the ${ }^{191} \mathrm{E}$ been highly preferred over $\mathrm{D}$ in this position of the prokaryotic RF1 loop? This may be from tuning giving a higher specificity for $A$ at the second position at the expense of efficiency (RF2 accommodates both A or G, whereas RF1 allows only A). A further increase in efficiency for the RF1-SPFD hybrid was obtained with the ${ }^{196} \mathrm{I}$-to-R substitution enhancing a marginally restored activity, but without converting to RF2 specificity or restoring the original RF1 specificity. This could be explained by potential hydrogen bonding between the introduced $\mathrm{R}$ and the phosphate of ${ }^{531} \mathrm{U}$ in the rRNA increasing loop stability in the recognition complex, or it may simply increase the factor's overall affinity for the ribosome, as found previously for charge-flip E-to-K mutations (Ito et al. 1998; Uno et al. 2002).

\section{Why have two decoding RFs been retained in eubacteria?}

Two specific RF1 anticodon loop variants recognized four codons, all three standard stop codons and the sense codon UGG (see Fig. 2). We can now deduce how RF1 and RF2 discriminate among the three stop codons and how they exclude the near-cognate codon, UGG. The RF1 loop and framework structure restricts recognition at the second position of the stop codon to A but allows both A and G to be recognized at the third position, whereas RF2 does the converse. An important consequence of this pattern is that recognition of UGG as a stop codon is excluded as neither factor allows recognition of $G$ at both the second and third positions.

This physiological recognition pattern breaks down in the two variants substituted at four or five sites in the recognition loop [RF1-SPF(D) and RF1-SPFD(ItoR)]. Residues important for determining the specificities of RF1 and RF2 at the second and third positions of the stop codon shown in Figure $5, \mathrm{~A}$ and $\mathrm{B}$, are deduced from the high-resolution structures. In RF1, the third amino acid in the PXT motif $\left({ }^{190} \mathrm{~T}\right)$ is critical for restricting recognition to $\mathrm{A}$ at the second position of the stop codon. In contrast, in RF2 the first amino acid in the SPF motif $\left({ }^{205} \mathrm{~S}\right)$ allows relaxed recognition of both $\mathrm{A}$ and $\mathrm{G}$ at this position. At the third position of the stop codon, ${ }^{185} \mathrm{Q}$ in strand $\beta 4$ of RF1 allows relaxed recognition of both $A$ and $G$, whereas in RF2 the equivalent ${ }^{202} \mathrm{~V}$ (perhaps with the involvement of ${ }^{213} \mathrm{R}$ ) restricts recognition to A only. From this analysis we deduce that the RF1-SPF(D) and RF1SPFD(ItoR) variants could recognize all four codons because, uniquely, they contain both the critical regions from the RF1 and RF2 loops that allow for relaxed recognition of either $A$ or $G$ at the second and third positions of the stop codon (see Fig. 5A). We inferred from our studies that the aspartic acid in SPFD is important for recognition of the second position of the stop codon as the RF1-SPF variant showed no 
A

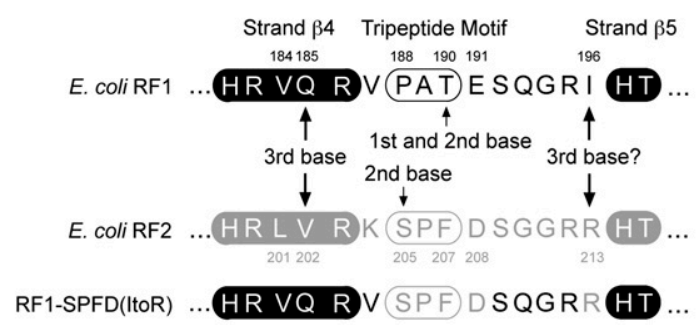

B

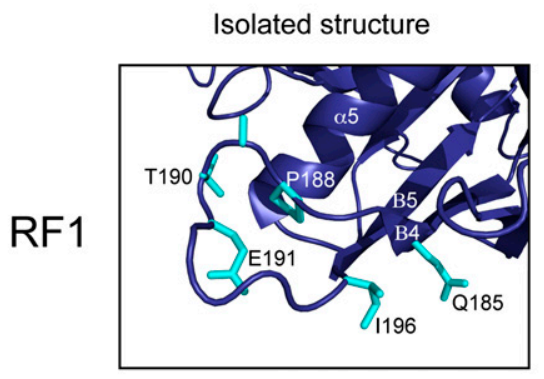

Ribosome bound structure
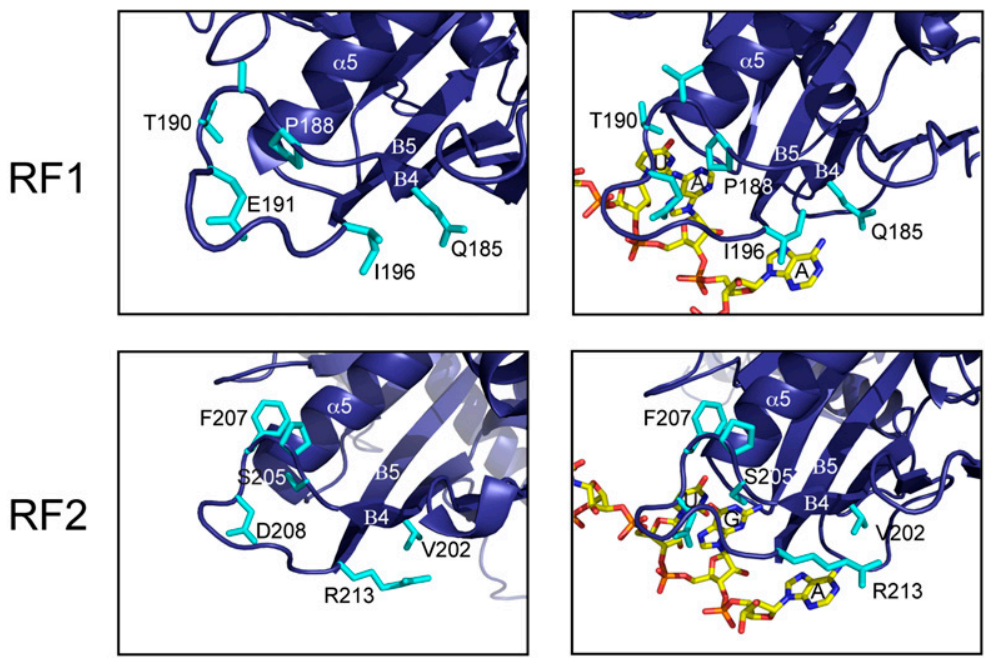

FIGURE 5. An explanation for how the recognition loops of RF1 and RF2 maintain specificity. (A) The recognition loop residues responsible for E. coli RF codon specificity. Residues that are important for determining the different specificities of RF1 and RF2 at the second and third positions of the stop codon are indicated. $(B)$ The isolated and ribosome-bound structures of the RF1 and RF2 recognition loops RF1 (Graille et al. 2005; Laurberg et al. 2008) and RF2 (Vestergaard et al. 2001; Weixlbaumer et al. 2008) with key elements highlighted (residues) or annotated (helices and strands).

functional activity in contrast to the RF1-SPF(D) variant (see Fig. 1C). This ${ }^{208} \mathrm{D}$, identified as a specificity-determining position (Oparina et al. 2005), must be critical either for loop orientation for residues that contact the stop codon or for packing the recognition loop onto the stop codon in the decoding center. From the structural arrangement of the eubacterial RFs, it should be possible to derive bacterial-like RFs that recognize a single codon (UAA), two codons, or four codons (including URR) as we have by chance created. Factors that recognize all four codons are probably excluded in biology because UGG is the only codon in the standard genetic code assigned to tryptophan.

\section{MATERIALS AND METHODS}

\section{Bioinformatic analysis}

Protein sequences were identified and aligned as described previously (Young et al. 2010).

\section{Bacterial strains}

Cloning utilized the E. coli strain DH5 $\alpha$ [supE44 DlacU169 (\$80lacZDM15) hsdR17 recA1 endA1 gyrA96 thi-1 relA1] (Hanahan 1983). Protein expressions were carried out in the E. coli strain BL21(DE3) pLysS [hsdS gal ( $\lambda$ cIts 857 ind1 Sam7 nin5 lacUV5.T7 gene 1) pLysS $\left(\mathrm{cam}^{R}\right)$ ] maintained in $30 \mu \mathrm{g} /$ $\mathrm{mL}$ chloramphenicol to retain the pLys plasmid (Studier et al. 1990). The 3A' translation termination assay studies used two $E$. coli $\mathrm{K}-12$ strains: the UAG suppressor strain $\mathrm{XA102}$ [ara $\operatorname{argE}$ (UAG) $\Delta$ (lacproB) nalA rif thi metB supE] and the UGA suppressor strain CDJ64 $[\Delta$ (lacproB) nalA rif sup9 valR thi] (Coulondre and Miller 1977).

\section{Vector construction and RF protein expression}

Construction of expression vectors used standard cloning and PCR mutagenesis protocols. pETRF1 and pTGRF1 construction has been described previously (Wilson et al. 2000; Mansell et al. 2001). Mutations to create variants were introduced into the RF1 prfA gene in both pETRF1 and pTGRF1 using a two-step PCR strategy (Sarkar and Sommer 1990) described earlier (Scarlett et al. 2003). Construction of the RF2 expression vectors pET21aRF2* T246A and pTGRF2* T246A has been described previously (Young et al. 2010). The RF2 expression vectors contain the RF2 $\operatorname{prfB}$ gene with a T246A mutation to counter a decrease in specific activity found upon overexpression of RF2 (Uno et al. 1996; Wilson et al. 2000). Supplemental Table 2 lists the PCR primer sequences used. The two-step PCR reactions were carried out as described (Young et al. 2010). In brief, step one used a mutagenic primer and a reverse primer to generate a mutated RF 3 ' product. Step two used a forward primer and purified step one RF $3^{\prime}$ product to amplify a variant full-length RF gene.

Protein expression and purification were carried out as described (Young et al. 2010). In brief, the RF proteins were expressed using standard protocols, purified by anion exchange (ResourceQ column; Amersham Pharmacia Biotech) before being subjected to dialysis and the protein concentration determined.

\section{The peptidyl-tRNA hydrolysis assay}

The in vitro hydrolysis assay was performed as described (Young et al. 2010) and measured $\mathrm{f}\left[{ }^{3} \mathrm{H}\right]$ Met release from the P-site tRNA in a complex comprised of MRE600 ribosomes, codons, and $\mathrm{f}\left[{ }^{3} \mathrm{H}\right]$ Met-tRNA ${ }^{\mathrm{fMet}}$ (Caskey et al. 1971). In brief, the substrate ( $\left[{ }^{3} \mathrm{H}\right]$ Met-tRNA ${ }^{\mathrm{fMet}}$.AUG-ribosome complex) was formed, incubated with RF and stop codon to induce hydrolysis, and then the reaction stopped. The $\left[{ }^{3} \mathrm{H}\right] \mathrm{fMet}$ released was extracted and measured in a scintillation counter. Data from an assay excluding 
RF were used to subtract background generated from nonspecific hydrolysis.

\section{The $3 \mathrm{~A}^{\prime}$ translation termination assay}

The $3 \mathrm{~A}^{\prime}$ assay uses a reporter protein comprising three repeats of an engineered derivative of the IgG-binding B domain (A' domain) of Staphylococcus aureus protein A (Moks et al. 1986; Nilsson et al. 1987) inserted into the vector pSM17 (MottaguiTabar et al. 1994). Two termination contexts, 5'-GAAACTTGA CTA-3' (Ra UGA Ra) and 5'-GATGGATAGCAT-3' (Ra UAG $\mathrm{Ra})$, were cloned between the second and third domains of the $3 \mathrm{~A}^{\prime}$ reporter protein as described (Cridge et al. 2006). E. coli strains containing cognate suppressor tRNAs (CDJ64, Ra UGA Ra; XA102, Ra UAG Ra), 3A' reporter and termination context (pSM17), and RF variants (pTG) were grown overnight [M9 media (+Trp) supplemented with $200 \mu \mathrm{g} / \mathrm{mL}$ ampicillin and $10 \mu \mathrm{g} / \mathrm{mL}$ gentamicin] at $37^{\circ} \mathrm{C}$. An aliquot $(500 \mu \mathrm{L})$ was then inoculated into a fresh culture [10 mL of M9 media (-Trp) and antibiotics] and $1 \mathrm{mM}$ isopropyl- $\beta$-D-thiogalactopyranoside $(25 \mu \mathrm{g} / \mathrm{mL})$ added to induce protein expression from the $3 \mathrm{~A}^{\prime}$ reporter construct (pSM17). The cultures were grown at $37^{\circ} \mathrm{C}$ until the $\mathrm{OD}_{600}$ reached 0.5 . Expression from the pTG vector (RF variants) was then induced with indoleacetic acid $(50 \mu \mathrm{L})$, and the cultures were grown for a further $3 \mathrm{~h}$ at $37^{\circ} \mathrm{C}$. Cells were chilled on ice $(15 \mathrm{~min})$ and pelleted ( $4000 \mathrm{~g}$ for $10 \mathrm{~min}$ at $4^{\circ} \mathrm{C}$ ), then lyzed prior to purification of the termination and readthrough proteins in IgG Sepharose minicolumns as previously described (Bjornsson et al. 1998). The eluted proteins were separated by $15 \%$ SDS-PAGE and visualized with Coomassie Brilliant Blue $\mathrm{R}$, and their intensities were determined by laser densitometry. As staining is proportional to the size of a protein, normalization of the three-domain readthrough product to the two-domain termination protein required adjustment by a factor of $2 / 3$ before the relative intensities of the termination and readthrough proteins could be used to calculate termination efficiencies using the formula: \% termination $=$ (termination band density)/(termination band density $+2 / 3$ readthrough band density).

\section{SUPPLEMENTAL DATA}

Supplemental material can be found at http://www.rnajournal.org.

\section{ACKNOWLEDGMENTS}

This work was supported by grants from the Marsden Fund of New Zealand (grant nos. UOO210 to W.P.T. and E.S.P, and UOO0701 to W.P.T.). We thank Sue Cutfield for helpful discussions.

Received February 3, 2010; accepted May 11, 2010.

\section{REFERENCES}

Askarian-Amiri ME, Pel HJ, Guevremont D, McCaughan KK, Poole ES, Sumpter VG, Tate WP. 2000. Functional characterization of yeast mitochondrial release factor 1. J Biol Chem 275: 1724117248.

Bjornsson A, Mottagui-Tabar S, Isaksson LA. 1998. The analysis of translational activity using a reporter gene constructed from repeats of an antibody-binding domain from protein A. Methods Mol Biol 77: 75-91.
Caskey CT, Beaudet AL, Scolnick EM, Rosman M. 1971. Hydrolysis of fMet-tRNA by peptidyl transferase. Proc Natl Acad Sci 68: 31633167.

Coulondre C, Miller JH. 1977. Genetic studies of the lac repressor. III. Additional correlation of mutational sites with specific amino acid residues. J Mol Biol 117: 525-567.

Crick FH. 1958. On protein synthesis. Symp Soc Exp Biol 12: 138-163.

Cridge AG, Major LL, Mahagaonkar AA, Poole ES, Isaksson LA, Tate WP. 2006. Comparison of characteristics and function of translation termination signals between and within prokaryotic and eukaryotic organisms. Nucleic Acids Res 34: 1959-1973.

Dale T, Uhlenbeck OC. 2005. Amino acid specificity in translation. Trends Biochem Sci 30: 659-665.

Freistroffer DV, Kwiatkowski M, Buckingham RH, Ehrenberg M. 2000. The accuracy of codon recognition by polypeptide release factors. Proc Natl Acad Sci 97: 2046-2051.

Frolova L, Le Goff X, Rasmussen HH, Cheperegin S, Drugeon G, Kress M, Arman I, Haenni AL, Celis JE, Philippe M, et al. 1994. A highly conserved eukaryotic protein family possessing properties of polypeptide chain release factor. Nature 372: 701-703.

Graille M, Heurgue-Hamard V, Champ S, Mora L, Scrima N, Ulryck N, van Tilbeurgh H, Buckingham RH. 2005. Molecular basis for bacterial class I release factor methylation by PrmC. Mol Cell 20: 917-927.

Hanahan D. 1983. Studies on transformation of Escherichia coli with plasmids. J Mol Biol 166: 557-580.

Ito K, Uno M, Nakamura Y. 1998. Single amino acid substitution in prokaryote polypeptide release factor 2 permits it to terminate translation at all three stop codons. Proc Natl Acad Sci 95: 81658169.

Ito K, Uno M, Nakamura Y. 2000. A tripeptide 'anticodon' deciphers stop codons in messenger RNA. Nature 403: 680-684.

Konecki DS, Aune KC, Tate W, Caskey CT. 1977. Characterization of reticulocyte release factor. J Biol Chem 252: 4514-4520.

Korostelev A, Asahara H, Lancaster L, Laurberg M, Hirschi A, Zhu J, Trakhanov S, Scott WG, Noller HF. 2008. Crystal structure of a translation termination complex formed with release factor RF2. Proc Natl Acad Sci 105: 19684-19689.

Laurberg M, Asahara H, Korostelev A, Zhu J, Trakhanov S, Noller HF. 2008. Structural basis for translation termination on the $70 \mathrm{~S}$ ribosome. Nature 454: 852-857.

Ledoux S, Uhlenbeck OC. 2008. Different aa-tRNAs are selected uniformly on the ribosome. Mol Cell 31: 114-123.

Mansell JB, Guevremont D, Poole ES, Tate WP. 2001. A dynamic competition between release factor 2 and the tRNA ${ }^{\mathrm{Sec}}$ decoding UGA at the recoding site of Escherichia coli formate dehydrogenase H. EMBO J 20: 7284-7293.

Moks T, Abrahmsen L, Nilsson B, Hellman U, Sjoquist J, Uhlen M. 1986. Staphylococcal protein A consists of five IgG-binding domains. Eur J Biochem 156: 637-643.

Mora L, Zavialov A, Ehrenberg M, Buckingham RH. 2003. Stop codon recognition and interactions with peptide release factor RF3 of truncated and chimeric RF1 and RF2 from Escherichia coli. Mol Microbiol 50: 1467-1476.

Mottagui-Tabar S, Bjornsson A, Isaksson LA. 1994. The second to last amino acid in the nascent peptide as a codon context determinant. EMBO J 13: 249-257.

Nakamura Y, Ito K. 2002. A tripeptide discriminator for stop codon recognition. FEBS Lett 514: 30-33.

Nilsson B, Moks T, Jansson B, Abrahmsen L, Elmblad A, Holmgren E, Henrichson C, Jones TA, Uhlen M. 1987. A synthetic IgG-binding domain based on staphylococcal protein A. Protein Eng 1: 107-113.

Okimoto R, Macfarlane JL, Clary DO, Wolstenholme DR. 1992. The mitochondrial genomes of two nematodes, Caenorhabditis elegans and Ascaris suum. Genetics 130: 471-498.

Oparina NJ, Kalinina OV, Gelfand MS, Kisselev LL. 2005. Common and specific amino acid residues in the prokaryotic polypeptide release factors RF1 and RF2: Possible functional implications. Nucleic Acids Res 33: 5226-5234. 
Pel HJ, Rep M, Grivell LA. 1992. Sequence comparison of new prokaryotic and mitochondrial members of the polypeptide chain release factor family predicts a five-domain model for release factor structure. Nucleic Acids Res 20: 4423-4428.

Petry S, Brodersen DE, Murphy FV, Dunham CM, Selmer M, Tarry MJ, Kelley AC, Ramakrishnan V. 2005. Crystal structures of the ribosome in complex with release factors RF1 and RF2 bound to a cognate stop codon. Cell 123: 1255-1266.

Petry S, Weixlbaumer A, Ramakrishnan V. 2008. The termination of translation. Curr Opin Struct Biol 18: 70-77.

Poole ES, Young DJ, Askarian-Amiri ME, Scarlett DJ, Tate WP. 2007. Accommodating the bacterial decoding release factor as an alien protein among the RNAs at the active site of the ribosome. Cell Res 17: 591-607.

Sarkar G, Sommer SS. 1990. The 'megaprimer' method of sitedirected mutagenesis. Biotechniques 8: 404-407.

Scarlett DJ, McCaughan KK, Wilson DN, Tate WP. 2003. Mapping functionally important motifs SPF and GGQ of the decoding release factor RF2 to the Escherichia coli ribosome by hydroxyl radical footprinting. Implications for macromolecular mimicry and structural changes in RF2. J Biol Chem 278: 15095-15104.

Scolnick E, Tompkins R, Caskey T, Nirenberg M. 1968. Release factors differing in specificity for terminator codons. Proc Natl Acad Sci 61: 768-774.

Shin DH, Brandsen J, Jancarik J, Yokota H, Kim R, Kim SH. 2004. Structural analyses of peptide release factor 1 from Thermotoga maritima reveal domain flexibility required for its interaction with the ribosome. J Mol Biol 341: 227-239.

Studier FW, Rosenberg AH, Dunn JJ, Dubendorff JW. 1990. Use of T7 RNA polymerase to direct expression of cloned genes. Methods Enzymol 185: 60-89.
Tate WP, Caskey CT. 1990. Termination of protein synthesis. In Ribosomes and protein synthesis: A practical approach (ed. G Spedding), pp. 81-100. Oxford University Press, Oxford, UK.

Uno M, Ito K, Nakamura Y. 1996. Functional specificity of amino acid at position 246 in the tRNA mimicry domain of bacterial release factor 2. Biochimie 78: 935-943.

Uno M, Ito K, Nakamura Y. 2002. Polypeptide release at sense and noncognate stop codons by localized charge-exchange alterations in translational release factors. Proc Natl Acad Sci 99: 1819-1824.

Vestergaard B, Van LB, Andersen GR, Nyborg J, Buckingham RH, Kjeldgaard M. 2001. Bacterial polypeptide release factor RF2 is structurally distinct from eukaryotic eRF1. Mol Cell 8: 1375-1382.

Weixlbaumer A, Jin H, Neubauer C, Voorhees RM, Petry S, Kelley AC, Ramakrishnan V. 2008. Insights into translational termination from the structure of RF2 bound to the ribosome. Science 322: 953-956.

Wilson DN, Guevremont D, Tate WP. 2000. The ribosomal binding and peptidyl-tRNA hydrolysis functions of Escherichia coli release factor 2 are linked through residue 246. RNA 6: 1704-1713.

Young DJ, Edgar CD, Murphy J, Fredebohm J, Poole ES, Tate WP. 2010. Bioinformatic, structural, and functional analyses support release factor-like MTRF1 as a protein able to decode nonstandard stop codons beginning with adenine in vertebrate mitochondria. RNA 16: 1146-1155.

Youngman EM, McDonald ME, Green R. 2008. Peptide release on the ribosome: Mechanism and implications for translational control. Annu Rev Microbiol 62: 353-373.

Zoldak G, Redecke L, Svergun DI, Konarev PV, Voertler CS, Dobbek H, Sedlak E, Sprinzl M. 2007. Release factors 2 from Escherichia coli and Thermus thermophilus: Structural, spectroscopic and microcalorimetric studies. Nucleic Acids Res 35: 1343-1353. 

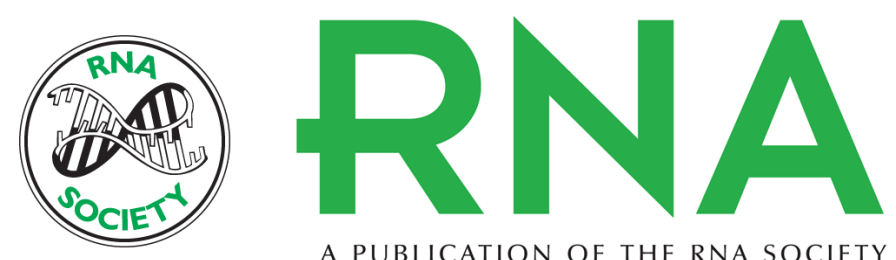

A PUBLICATION OF THE RNA SOCIETY

\section{The codon specificity of eubacterial release factors is determined by the sequence and size of the recognition loop}

David J. Young, Christina D. Edgar, Elizabeth S. Poole, et al.

RNA 2010 16: 1623-1633 originally published online June 28, 2010

Access the most recent version at doi:10.1261/rna.2117010

Supplemental
Material http://rnajournal.cshlp.org/content/suppl/2010/06/08/rna.2117010.DC1

References This article cites 42 articles, 12 of which can be accessed free at: http://rnajournal.cshlp.org/content/16/8/1623.full.html\#ref-list-1

License

Email Alerting Receive free email alerts when new articles cite this article - sign up in the box at the Service top right corner of the article or click here. 